\title{
Establishment of an in vitro evaluation system for drug-coated balloons
}

\author{
Kaule $\mathrm{S}^{1}$, Petersen $\mathrm{S}^{1}$, Minrath $\mathrm{I}^{1}$, Schmitz K-P ${ }^{1}$, Sternberg $\mathrm{K}^{1}$ \\ ${ }^{1}$ Institute for Biomedical Engineering, University of Rostock, Germany
}

sebastian.kaule@uni-rostock.de

\begin{abstract}
The drug-coated balloon (DCB) is an emerging device in percutaneous coronary intervention, which has shown promising results by means of an efficient local release of paclitaxel (PTX) without the need of an implant remaining in the patient's body. Safety and efficacy of $D C B$ have been already proven in clinical trials for the treatment of in-stent restenosis and complex coronary de novo lesion subsets, such as small vessel diseases, diabetes, and diffuse lesions, where stents obtain suboptimal results. However, there is only very few literature data about coating and drug delivery characteristics. In this context, we set up an in vitro evaluation system for $D C B$ including determination of coating morphology, coating thickness, mechanical performance, drug loss and drug application as well as particle release during simulated use. Exemplary data of drug delivery characteristics of an urea-based DCB are provided within the present paper.
\end{abstract}

\section{Keywords: drug-delivery, drug- coated balloons, implant coating}

\section{Introduction}

In recent years drug-coated balloons (DCB) providing a short-term transfer of the antiproliferative drug paclitaxel (PTX) to the arterial wall have emerged as a therapeutic alternative to drug-eluting stents in vascular intervention. In fact they should reduce the complication of in-stent restenosis and their safety and efficacy have been proven in different clinical trials [1]. However, the development of DCB is complex as the coating should be robust enough to physically maintain the drug on the surface of the balloon during transit of the device through the vascular system while still allowing a rapid, uniform and efficient drug transfer to the vessel wall during balloon dilatation. Although a lot of clinical data led to CE-approval of different DCB, only very few in vitro data regarding coating and drug delivery characteristics can be found in literature. For example, while Kelsch et.al. determined percental drug loss ratios for DCB during in vitro passage through a haemostatic valve and a guiding catheter [2], the correlation of coating composition with DCB performance is not discussed. Such in vitro studies should be however considered as highly important for the understanding of the DCB coating requirements, the resulting drug delivery and hence the improvement of established DCB. In this regard, we established an in vitro evaluation system considering the specific requirements and challenges of DCB such as coating morphology, coating thickness, drug loss and transfer to the vessel wall, resid- ual drug load on the balloon surface as well as particle release during simulated use in an in vitro vessel model and deliverability defined by the functional parameters trackability and crossability (Fig. 1).

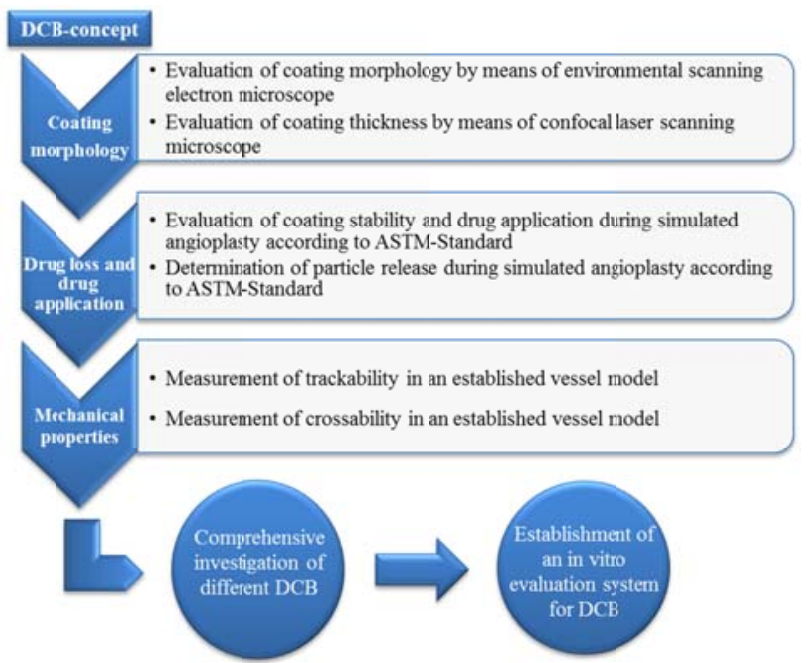

Figure 1: In vitro evaluation system for DCB

In the following we provide exemplary data for the drug delivery characteristics of an urea-based DCB during simulated use in an in vitro vessel model.

\section{Methods}

Simulated angioplasty in an in-vitro vessel model: Simulation of a coronary balloon angioplasty was performed according to ASTM F2394-07, X.2.4 in an invitro vessel model. The model, described in literature [3], consists of a guiding catheter (Cordis Vista brite tip 5F JL3.5, I.D. 0,056“, REF 556-002-0L, Cordis Corporation (J\&J) Warren/Bridgewater Campus, Massachusetts, USA), a guide wire (BIOTRONIK Galeo M 0,014“, REF 114156, BIOTRONIK AG, Bülach, Switzerland) and a tortuous path milled in two PMMA-plates and revetted with a PTFE tube (BIOTRONIK AG, Bülach Switzerland). The whole model was surrounded by $37 \pm 2{ }^{\circ} \mathrm{C}$ heated water. At the distal end of the test path we placed a model stenotic vessel (silicone tube, $\mathrm{di}=4 \mathrm{~mm}$, according to outer diameter of dilated DCB). Firstly, after advancing the DCB over the guide wire through the guiding catheter into the vessel model, we flushed the model with $27 \mathrm{ml}$ $0.09 \% \mathrm{NaCl}$ to recover drug lost during transit through the system ("after track 1"). Secondly, we inflated the balloon to nominal pressure (7 atm) and held it for 30 seconds. Afterwards we deflated the balloon, collected the 
solution contained in the silicone tube and filled it up to $27 \mathrm{ml} 0.9 \% \mathrm{NaCl}$ ("after dilatation 1"). In the next step the silicone tube was stored in $10 \mathrm{ml}$ methanol for $30 \mathrm{~min}$ for extraction ("after dilatation 2). Finally we cut of the balloon and stored it in an empty vial for determination of residual drug concentration on the balloon (extraction for $30 \mathrm{~min}$ in $10 \mathrm{ml}$ methanol, "residual drug-load"). As a last step we flushed again the entire system with $27 \mathrm{ml}$ methanol to determine the residual PTX content released during transit of the DCB ("after track 2") and prepared the model for the next test sample by flushing the system with $50 \mathrm{ml} 0.9 \% \mathrm{NaCl}$.

Measurement of drug-load,-loss and -transfer during simulated use: We examined the drug content in every test solution ("after track 1", "after track 2", "after dilatation 1", "after dilatation 2", "residual drug load") by means of HPLC after 1:10 dilution with methanol. Furthermore we summed up the results of the measurements after track 1 and 2 and after dilatation 1 and 2 to present them as one result for drug loss during transit and drug transfer in the silicone tube respectively. In conclusion the total drug load is the sum of all measured PTX amounts.

HPLC parameters: $20 \mu \mathrm{L}$ of the test solutions were injected into an Eurospher column 100-5, C18, 120 x 4 mm ID (Wissenschaftlicher Gerätebau Dr.-Ing. Herbert Knauer GmbH, Berlin, Germany). The chromatographic conditions were: column temperature $23{ }^{\circ} \mathrm{C}$, isocratic eluent PBS (0.005 M, pH 3.5) - acetonitrile 50 - 50\% $(\mathrm{v} / \mathrm{v})$, flow rate $1.0 \mathrm{~mL} / \mathrm{min}$ and $\mathrm{UV}$ detection at $230 \mathrm{~nm}$ with calibrated measurement range $0.5-20.0 \mathrm{mg} / \mathrm{L}$ and detection limit approximately $0.05 \mathrm{mg} / \mathrm{L}$.

\section{Results}

For the evaluation of the in vitro efficacy concerning drug loss, drug application, residual drug load and entire drug concentration on the balloon we determined an urea-based DCB. Representative results of the DCB after simulated use in the in-vitro vessel model (ASTM F2394-07, X.2.4) can be seen in Figure 2. During tracking the balloon lost $58.22 \%$ of its entire drug load of $3.49 \mu \mathrm{g} / \mathrm{mm}^{2} .6 .61 \%$ of the entire drug load could be placed in the silicone tube while $35.17 \%$ remained on the balloon after dilatation.

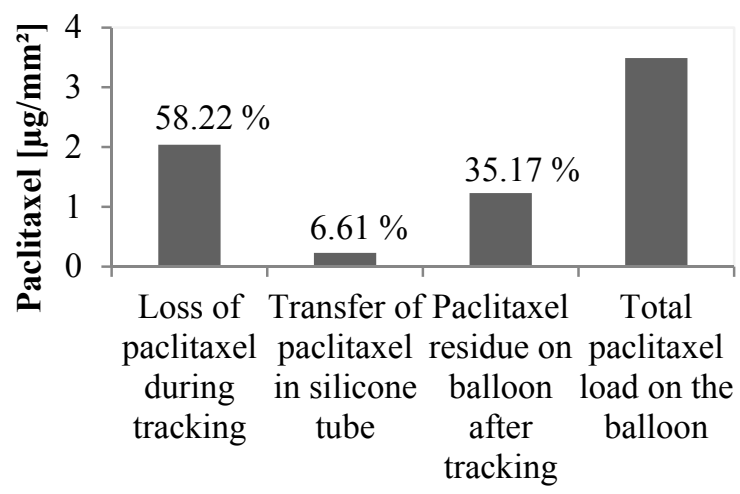

Figure 1: Comparison of absolute drug loss, drug transfer and drug residue of an urea-based DCB after simulated use in an in vitro vessel model (ASTM F2394-07, X.2.4).

\section{Discussion}

Although this work is more a description of different methods for in vitro characterization of DCB, we will briefly discuss the given results. Due to the high water solubility of urea and the mechanical stress during the passage of the balloon through the tortuous path of the vessel model, the drug loss during transit of the balloon is very high. In consequence the transfer of PTX in the silicone tube decreased and only a barely percentage of the entire load could be placed in the silicone tube. These results were in the same range as before mentioned results of Kelsch et.al. as they give percental drug losses of $26 \%-42 \%$ while advancing the DCB through a haemostatic valve and a guiding catheter [2]. Although the results might not directly correlate with the in vivo performance of the DCB, the described model is an advancement to the simple test procedures described in literature. One could hence assume that well performing DCB in the vessel model according to ASTM-Standard will show promising results in animal studies or other in vivo investigations.

\section{Conclusion}

To summarize, we examined an urea-based DCB-concept and evaluated it in the grading system (data not shown). Furthermore we established different methods for characterising DCB-concepts concerning their in-vitro efficacy and for a better understanding of their function. We will now aim for a comprehensive study to determine different DCB to specify their limitations and improve current concepts.

\section{Acknowledgement}

We would like to thank Thomas Reske, Martina Schröder, Christopher Schwerdt for their expert technical assistance and the Biotronik SE \& Co. KG, Erlangen, Germany for the generous supply of the uncoated balloon catheter. Furthermore, financial support by Bundesministerium für Bildung und Forschung (BMBF) within REMEDIS "Höhere Lebensqualität durch neuartige Mikroimplantate" (FKZ: 03IS2081) is gratefully acknowledged.

\section{Bibliography}

[1] Waksman, J.P., Loh, R.: Paclitaxel Drug-Coated Balloons - A Review of Current Status and Emerging Applications in Native Coronary Artery De Novo Lesions, J Am Coll Cardiol Intv., vol. 5, pp. 1001-1012, October 2012

[2] Kelsch, B., Scheller, B., et.al.: Dose Response to Paclitaxel-Coated Balloon Catheters in the Porcine Coronary Overstretch and Stent Implantation Model, Invest Radiol, vol. 46, pp. 255-263, April 2011

[3] W. Schmidt, "Instrumentation", in Catheter-Based Cardiovascular Interventions: A Knowledge-Based Approach (P. Lanzer, edt.), ch. 27, pp. 445-471, Heidelberg, Berlin, Berlin: Springer, 2013 